\title{
Analysis of Hybrid Parameters of a Single Stage Small Signal Transistor Amplifier Using Two-Port Network
}

\author{
Henry Erialuode Amhenrior \\ Department of Electrical/Electronic Engineering, Faculty of Engineering, University of Benin, Benin City, Nigeria
}

Email address:

henrino2003@yahoo.com

To cite this article:

Henry Erialuode Amhenrior. Analysis of Hybrid Parameters of a Single Stage Small Signal Transistor Amplifier Using Two-Port Network. Nanoscience and Nanometrology. Vol. 4, No. 1, 2018, pp. 9-15. doi: 10.11648/j.nsnm.20180401.12

Received: July 19, 2018; Accepted: August 3, 2018; Published: August 31, 2018

\begin{abstract}
This paper presents the analysis of the h-parameter of a single stage small signal transistor amplifier using twoport network. This is motivated by the need to have the rudimentary knowledge of the h-parameter transistor analysis model and to demonstrate its equivalence in the three configuration of the transistor. In this analysis, it was borne in mind the characteristics of a small signal operating conditions of the transistor. Among these characteristics is the assumption that Small - signal operations applies when variations are restricted to such a small amplitude that the partial differential derivatives can be applied. In the methodology, the transistor two-port network was used in deriving the hybrid parameter performance quantities from the first principle by applying partial derivative mathematical tool. The four variables of the input and output of the two-port network namely the currents and voltages which could be combined in six different ways were identified. These were described and analyzed by mathematical functions which relates one of the four variables to two other variables at a time, and by this, six various functions were generated. The partial derivatives of these functions were obtained and these gave the performance quantities which are the key components of the $\mathrm{h}$ parameter equations. The initial conditions of each of the component parameters where properly observed and substituted appropriately. This was then transformed into the equivalent circuits of the three transistor configurations. Since the transistor is frequently used for the amplification of sinusoidal currents, the changes in instantaneous total voltage and current were then replaced by root mean square ac quantities, which are considered to be impressed on the direct value. The results obtained are the basic h-parameter equations and the key components of the $\mathrm{h}$ parameter transistor model namely the Input Resistance, the Reverse Voltage Gain, the Forward Current Gain and the Output Admittance.
\end{abstract}

Keywords: H-Parameter, Two-Port Network, Performance Quantities, Equivalent Circuits

\section{Introduction}

The most important parameters of transistor amplifiers are the gains and the matching impedances. The gains of a transistor circuit could be derived graphically by plotting the load lines on the output characteristics. But this is not truly a reliable practical method and most datasheet from manufacturers do not contain graphs. Again, it is a time consuming method of analysis [1]. In order to avoid these problems, models are usually used to predict the behavior of transistors more appropriately in the region of operations of the transistor. Transistors can be analyzed using a number of models namely the Z-parameters, Y-parameters, and the hybrid model (h-parameters) [1].

In a small signal ac transistor amplifier usually with small amplitude, two models are commonly in use: the hybrid model and $r_{e}$ model [2]. The h-parameter is used in the analysis of a small signal transistor to derive the gains and the impedances. This model is appropriate for small signals at middle band and represents the activity of the transistor [2]. The hybrid model is commonly used because the parameters are real numbers at audio frequencies; they are easy to measure; they can be gotten from the transistor static characteristic curve; the parameters are easy to use in analyzing circuit and design and they are usually specified by the manufacturers [3].

The use of the model could be made more efficient by having a proper understanding of the construction of the model and most users of h-parameter do not have the rudimentary mathematical grasping of this model. Hence, 
this study seeks to investigate and analyze the h-parameter model of a single stage small signal amplifier using mathematical tools from first principle to aid the proper analysis of transistor amplifiers. In this analysis therefore, transistor as a two-port network will be considered and mathematical partial derivative as a tool for the realization of the transistor gains and impedances which are the basic units of the small signal h-parameter analysis.

\section{Literature Review}

In comparison with other animals and forces of nature, human beings are weak physically. In a like manner, most signals are usually weak for some applications. It is on the realization of this that the need to develop a device which can harness energy so that very little physical ability is needed to perform different task arose [4]. This device was called an amplifier. Signals and energies get weakened as they are transmitted along their channels due to attenuation, interference, distortion, magnetic effect etc. As a result, these signals and energy usually need amplifiers to retain their strength. A very efficient amplifier for small signals are the transistors.

The performance of the transistor depends among other things on the analysis of its configuration. As a result, many scientists, authors and researchers have evolved various ways to analyze the transistor amplifier over the years leading to the development of transistor equivalent circuits. According to some authors, two prominent positions regarding the equivalent circuit to be used for a transistor are common today namely the h-parameter model and the $r_{e}$ model [5]. For some period of time, both industrial and educational institutions relied heavily on the h-parameter model. The hybrid parameter equivalent circuit continues to be very popular, notwithstanding the existence of an equivalent circuit obtained rightly from the operating conditions of the transistor - the $r_{e}$ model. Makers of transistors keep specifying the hybrid parameters for a particular operating region on the datasheets. The parameters of the model can be gotten straightly from the hybrid parameters in this region. However, the hybrid equivalent circuit has a setback of being restricted to a distinct set of working situation for it to be seen to be accurate. The parameters of the other equivalent circuits can be found for any region of operation within the active region and are not restricted by the single set of parameters provided by the data sheet. In turn, however, the $r_{e}$ model fails to account for the output impedance level of the device and the feedback effect from output to input. In addition, some author have opined that $r_{e}$ model is the easiest to understand [6].

Two-port model has been used severally to analyze electronic devices. Lobna proposed a two port network three impedance oscillators analyzed and all the attainable realizations using various arrangements. They studied the results of each arrangement on the oscillation condition and the frequency of oscillation. They then generate the transmission matrices for small-signal equivalent models of the BJT and MOS transistors [7]. Ahmed and Mohammad modeled all the feasible four-impedance settings that give a good second-order two-stage Colpitts oscillator using twoport network transmission parameters, and this they tested by the use of BJT and MOS transistors in an experimental case [8]. However, Ahmed addressed the task of Ahmed and Mohammad above for BJT and MOS in favour of two-port network transmission parameters for general design equations not limited to matched devices [9]. In another study, Ahmed proposed two instances of two-port analysis namely, an elementary and the advanced; he derived the transmission matrices for small-signal equivalent models of the BJT and MOS transistors. He suggested that his derived two-port network is especially good to employ in network analysis because the expression is only derived once and is independent of the complexity of any transistor. Again, it uses inter-network connectivity in which network could be divided into series, parallel or cascade interconnects [10].

In this approach, the h-parameter will be analyzed as a two-port network from first principle using some mathematical tools and then transform into its equivalent circuits of the three transistor configuration network.

\subsection{History of Transistor}

In 1904, the vacuum tube diode was introduced by J. A. Fleming [5]. The development of the transistor started its course from the vacuum tube. Thomas Edison used the application of vacuum tube to produce the Edison light bulb [11]. This discovery led to more researches by adding more control grid to the vacuum tube. As a result, Lee De Forest in 1906, added a third element called the control grid, to the vacuum diode resulting in the first amplifier, the triode. In the early 1930s the four-element tetrode and five-element pentode gained prominence in the tube industries [5].

On December 23, 1947 the amplifying action of the first transistor was successfully demonstrated at Bell Laboratories in Murray Hill, New Jersey. The three individuals credited with the invention of the transistor were William Shockley, John Bardeen and Walter Brattain. Shockley had started working in 1936 on the solid state physics theory that was the basis for the transistor [12]. This three-terminal solid-state device has several advantages over the tubes namely, small size and light weight; it had no heat requirement or heat loss; it had a very strong construction; it was more efficient as less power was absolved for its own operation; there was no warm-up period required; and lower operating voltage was possible [5].

What appears to be the birth of modern transistor came in April 1954 when Adcock, Teal, and their team used a high purity silicon to fabricate an npn structure that has an emitter region that is carefully doped with impurity to increase current gain and a base layer thickness of 25 micrometers made of $\mathrm{p}$-type material [13]. Also in 1954, Texas Instruments of Dallas, Texas commenced the commercial manufacturing of junction transistors for use in small radios, while in 1960, The Sony Company of Japan started the production of television sets from transistors after obtaining 
the right to produce transistors [12].

The transistor is still undergoing changes and improvements in its operations and applications through the efforts of researchers. Recently, Jose reported that a group of scientists had made a transistor that is able to mimic "some characteristics of neurons, such as counting, remembering and performing simple arithmetic operations [14].”

\subsection{Transistor Operation}

The transistor can be conventionally be represented as shown in Figure 1, while Figures 2 and 3 will help to explain the operation of a transistor.

When the emitter-base junction is forward biased, the emitter sends large number of electrons into the base region. Only few of the emitter carriers will combine with the holes in the base material because the base does not contain a great number of holes. Most of the emitter carriers will come under the influence of the collector field and be swept through the depletion region and into the collector. The collector is positive with respect to the base and will attract any electron it can get. It is a statistical process whereby the electrons are far less likely to combine with holes and become base current than they are likely to be swept into the collector. Typically, only 1 or 2 percent of the emitter current become base current, the balance 99 or 98 percent become collector current [15].

The operation of the PNP transistor shown in Figure 3 is exactly the same as that of NPN if the roles played by the electrons and holes are interchanged and the junctions reverse biased. But the flow of holes in the PNP transistor is slower than the flow of electron in the NPN transistor. For this reason NPN transistors are better choices for high frequency applications.

In summary, the base current is a small percentage of the total current. The emitter current is the total current $\left(I_{E}=\right.$ $\left.I_{C}+I_{B}\right)$ and the collector current is almost as large. The base current controls the emitter and collector currents. The NPN and PNP transistor are electrical complements.

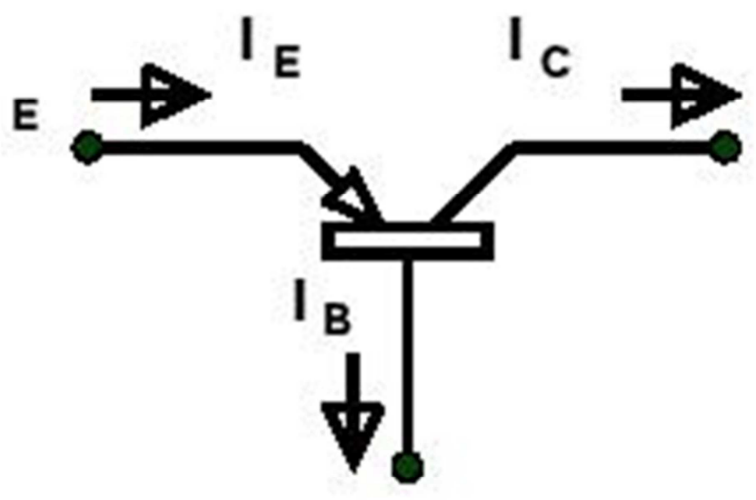

B

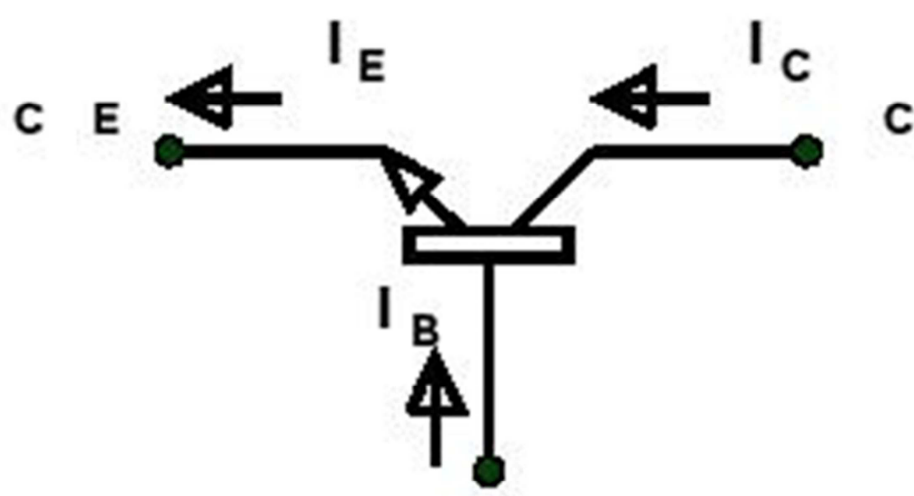

B

Figure 1. (a) PNP Transistor Conventional Representation (b) NPN Transistor Conventional Representation.

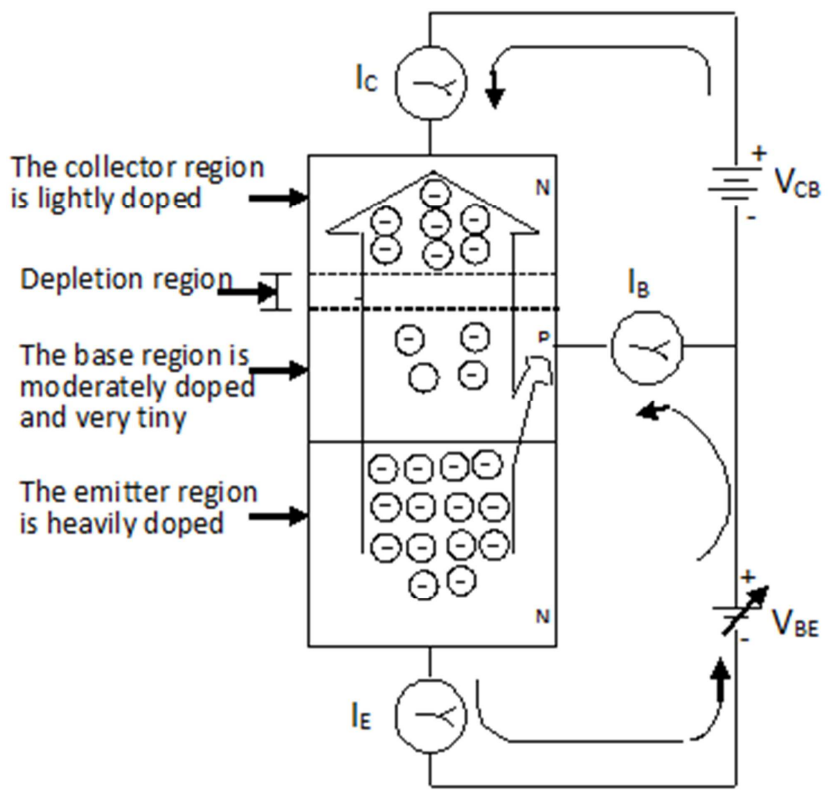

Figure 2. NPN Transistor Current in Circuit Operations [15].

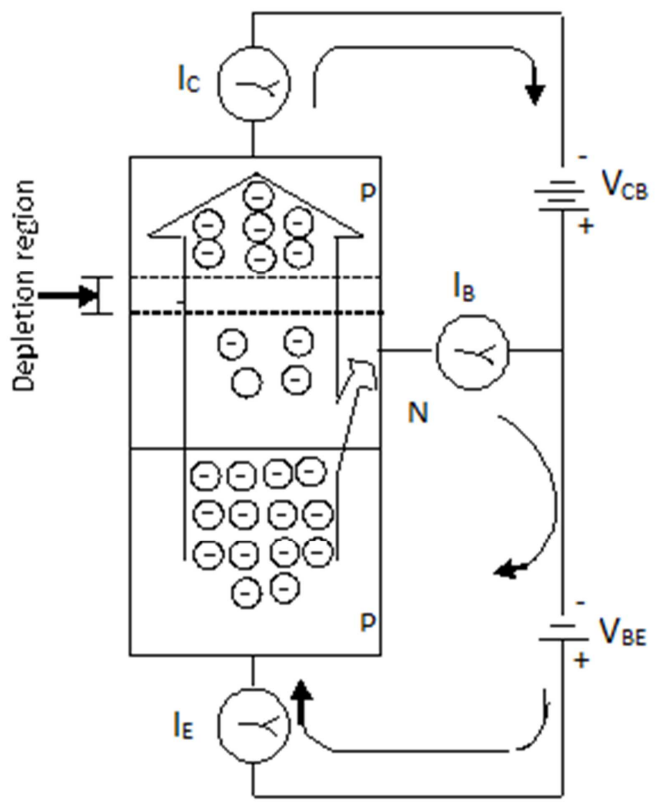

Figure 3. PNP Transistor Current in Circuit Operations [15] 


\subsection{The Transistor as a Two-Port Device and Alternating Current Operations}

The transistor is mainly used as an amplifier of alternating currents, but suitable direct current operating condition must be established. Any one of its three conditions can be regarded as a two-part device linking the input and output circuits. The general representation of transistor as a two-port device is as shown in figure 4 below.

The symbols used in the figures define the instantaneous total current and voltage in the input and output terminals.

There are four variables in the two-port representation of the device given above. These are the two voltages $V_{1}$ and $V_{2}$ and two currents $i_{1}$ and $i_{2}$. For ac analysis, the mathematical relationship between the four variables is better considered. In general, an amplifier is operated in the region where the characteristic curves are straight lines so that the equations should be linear. In this paper, the common emitter configuration is used for analysis. The output characteristics are a plot of the output current $I_{C}$ versus output voltage $V_{C E}$ for a range of values of input current $I_{B}$. The input characteristics are a plot of the input current $I_{B}$ versus the input voltage $V_{B E}$ for a range of values of output voltage $V_{C E}$ $[5,15]$. Figure 5 shows the input and output characteristics curves respectively.

\section{Methodology}

In this section, the derivation of the performance quantities was carried out from the first principle using among other things the mathematical tool of partial differential equation and the results were used to obtain the basic equations of the h-parameters relating the basic inputs and outputs of currents and voltages of the transistor as a two-port network.

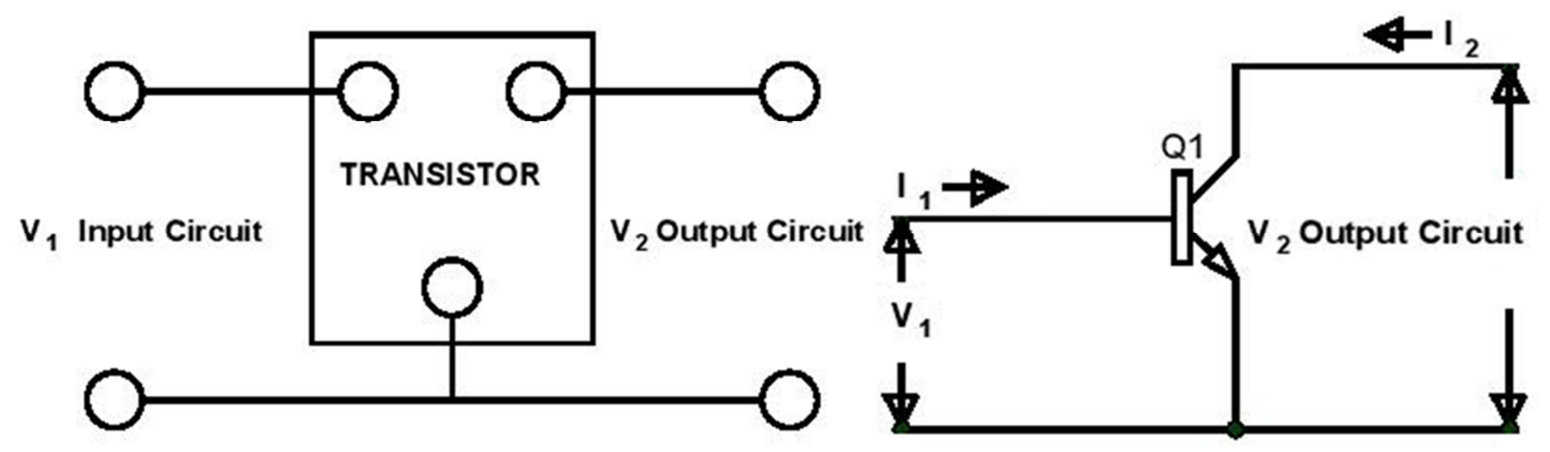

Figure 4. (a) General Two-port network representation (b) Two-port representation of an NPN Transistor.
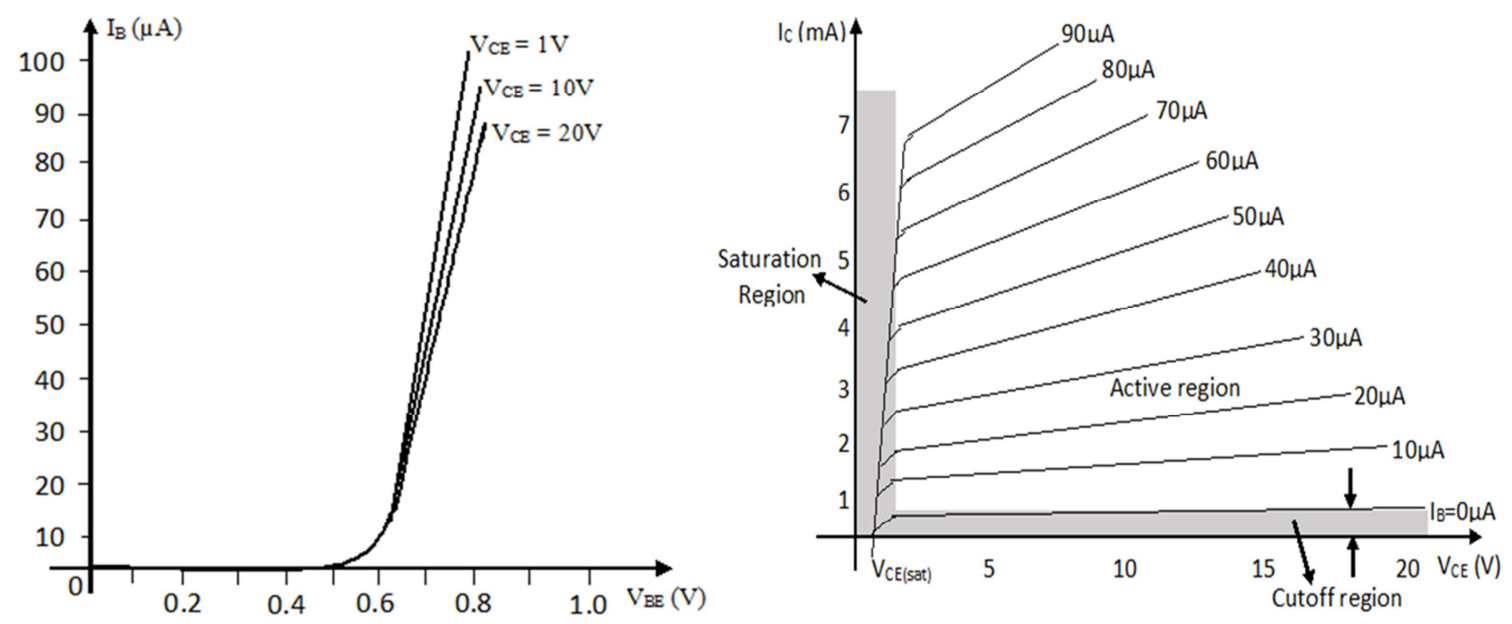

Figure 5. (a) Input Characteristics of CE configuration [5] (b) Output Characteristics of CE configuration [5].

\subsection{Mathematical Analysis of Transistor Using H-Parameters}

Assumptions:

The basic assumption in arriving at a transistor linear model or equivalent circuit is that the variations about the quiescent points are assumed to be small, so that the transistor parameters can be considered over the signal excursion.

Considering the two-port network shown in Figure 4 and represented by a common-emitter transistor configuration, the symbols used in the figure defines the instantaneous total current and voltage at the input and output terminals. There are four variables depicting these input and output of the system which are

$$
v_{1}=v_{b e}, i_{1}=i_{b}, v_{2}=v_{c e}, i_{2}=i_{c}
$$

Two of these four variable can be selected as independent variable leaving the other two as dependent variable in 
$C_{4}^{2}=6$ ways [16].

If $F_{a}$ and $F_{b}$ are some of the functions relating one of these variables say $V_{l}$ with the other three variables $\left(V_{2}, i_{1}, I_{2}\right)$, we can write thus,

$$
V_{1}=F_{a}\left(V_{2}, i_{1}, I_{2}\right)
$$

Similarly, the quantity $V_{2}$, with the function $\mathrm{F}_{\mathrm{b}}$ involving $V_{l}$, $i_{1}$, and $I_{2}$ could be given as:

$$
V_{2}=F_{b}\left(V_{1}, i_{1}, I_{2}\right)
$$

From (1) and (2),

$$
\begin{aligned}
V_{1} & =F_{1}\left(i_{1}, I_{2}\right) \\
V_{2} & =F_{2}\left(i_{1}, I_{2}\right)
\end{aligned}
$$

Where $F_{1}$ and $F_{2}$ are functions which can be found if the function $\mathrm{F}_{\mathrm{a}}$ and $\mathrm{F}_{\mathrm{b}}$ are known.

Equations (3) and (4) shows that $i_{1}$ and $i_{2}$ can be considered as independent variables.

By similar analysis to the above, the two currents can be written in terms of the two voltages as

$$
\begin{gathered}
i_{1}=F_{3}\left(V_{1}, V_{2}\right) \\
i_{2}=F_{4}\left(V_{1}, V_{2}\right)
\end{gathered}
$$

From the input-output characteristic curve of the common emitter transistor shown in Figure 5, the following can be deduced.

$$
\begin{aligned}
& V_{1}=F_{5}\left(i_{1}, V_{2}\right) \\
& i_{2}=F_{6}\left(i_{1}, V_{2}\right)
\end{aligned}
$$

These relations apply to the instantaneous total voltages and currents.

For small variations in these quantities above the operating point, (3) and (4) becomes

$$
\begin{aligned}
& \delta V_{1}=\frac{\partial V_{1}}{\partial i 1} \delta_{i 1}+\frac{\partial V_{1}}{\partial_{i 2}} \delta_{i 2}[11] \\
& \delta V_{2}=\frac{\partial V_{2}}{\partial_{i 1}} \delta_{i 1}+\frac{\partial V_{2}}{\partial_{i 2}} \delta_{i 2}[11]
\end{aligned}
$$

Small - signal operations applies when variations are restricted to such a small amplitude that the partial differential terms in equations (9) and (10) can be considered as constant.

The four partial differential terms have the dimension of impedance and can be written as

$$
\begin{aligned}
& Z_{11}=\frac{\partial V_{1}}{\partial_{i 1}} ; Z_{12}=\frac{\partial V_{1}}{\partial_{i 2}} \\
& Z_{21}=\frac{\partial V_{2}}{\partial_{i 1}} ; Z_{22}=\frac{\partial V_{2}}{\partial_{i 2}}
\end{aligned}
$$

Similarly, from equations (7) and (8), the four partial differential terms have hybrid or h-parameters and can be written as

$$
\begin{gathered}
\delta V_{1}=\frac{\partial V_{1}}{\partial_{i 1}} \delta_{i 1}+\frac{\partial V_{1}}{\partial_{V 2}} \delta_{V 2} \\
\delta i_{2}=\frac{\partial i_{2}}{\partial_{i 1}} \delta_{i 1}+\frac{\partial i_{2}}{\partial_{V 2}} \delta_{V 2}
\end{gathered}
$$

Where

$$
\begin{aligned}
& h_{11}=\frac{\partial V_{1}}{\partial i_{1}}(\mathrm{ohms}) ; h_{12}=\frac{\partial V_{1}}{\partial V_{2}} \text { (no dimension) } \\
& h_{21}=\frac{\partial i_{2}}{\partial i_{1}}(\text { no dimension }) ; h_{22}=\frac{\partial i_{2}}{\partial V_{2}}(\text { mhos })
\end{aligned}
$$

Normally, the transistor will frequently be used for the amplification of sinusoidal currents. Hence it is convenient to replace the changes in the instantaneous total voltage and current by root mean square ac quantities, which are considered to be impressed on the direct value.

Equations (15) and (16) may now be written as

$$
\begin{gathered}
V_{1}=h_{11} I_{1}+h_{12} V_{2} \\
I_{2}=h_{21} I_{1}+h_{22} V_{2}
\end{gathered}
$$

Where the four generalized $\mathrm{h}$ - parameters are defined by the following expressions.

1. Input impedance with the output terminals short circuited is defined by

$$
h_{11}=\frac{V_{1}}{I_{1}} \text { with } \mathrm{V}_{2}=0
$$

2. Reciprocal of the voltage gain with input terminals open circuited is defined by

$$
h_{12}=\frac{V_{1}}{V_{2}} \text { with } \mathrm{I}_{1}=0
$$

3. Current gain are with the output terminals short circuited is defined by

$$
h_{21}=\frac{I_{2}}{I_{1}} \text { with } \mathrm{V}_{2}=0
$$

4. Reciprocal of the output impedance with the input terminals open circuited is defined by

$$
h_{22}=\frac{I_{2}}{V_{2}} \text { with } \mathrm{I}_{1}=0
$$

Where $\mathrm{h}_{11}$ is impedance, $\mathrm{h}_{22}$ is admittance, and $\mathrm{h}_{12}$ and $\mathrm{h}_{12}$ are gains and are non-dimensional.

\subsection{Equivalent Circuits Using H-Parameters}

The h- parameters are defined in technical literature for all three transistors circuit connections: common-emitters, common-base, and common-collector. Different subscripts are used for each circuit and the first subscript defines the transistor circuit configuration for which the $\mathrm{h}$ - parameter is used. The various configurations are as shown below using NPN transistor in Figures $6-8$. 


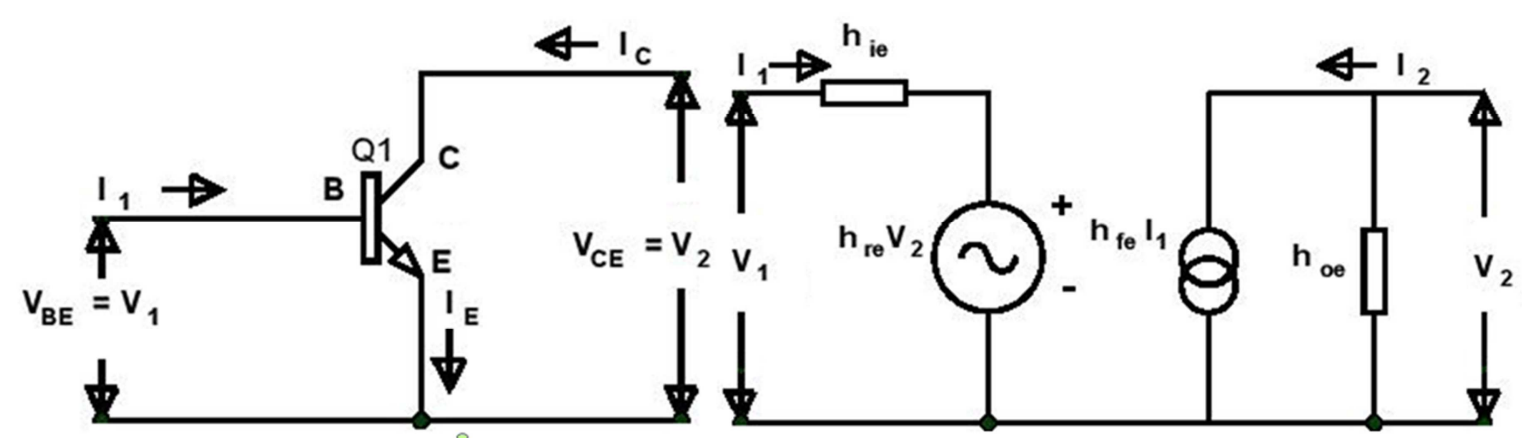

Figure 6. (a) Common-emitter configuration (b) h-parameter equivalent circuit of a common-emitter config.

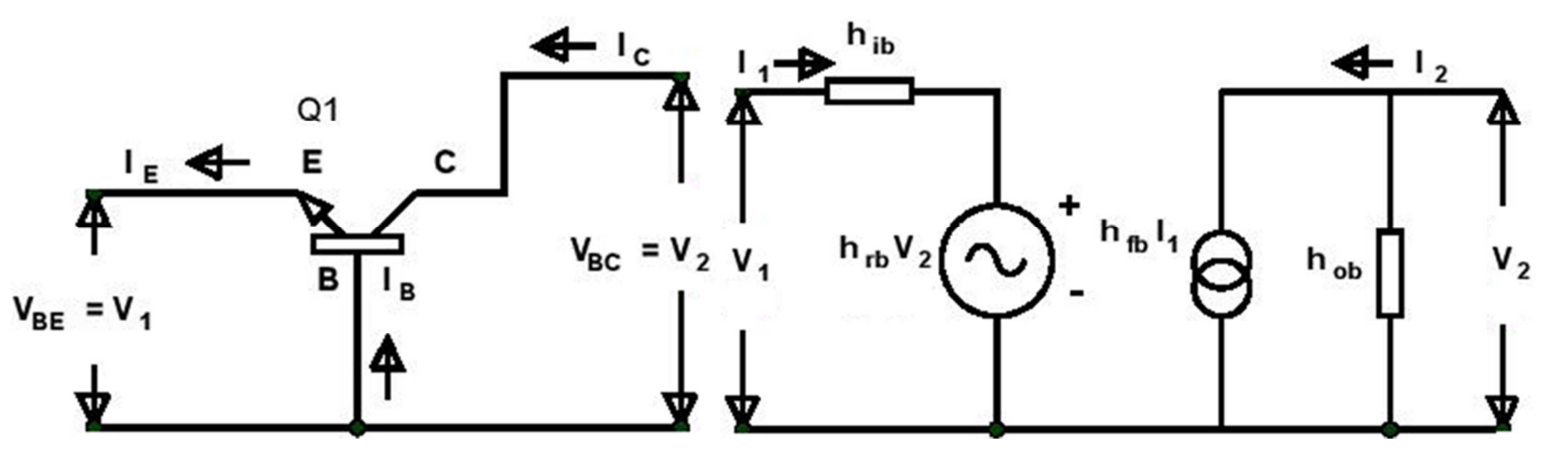

Figure 7. (a) Common-base configuration (b) h-parameter equivalent circuit of a common-base config.

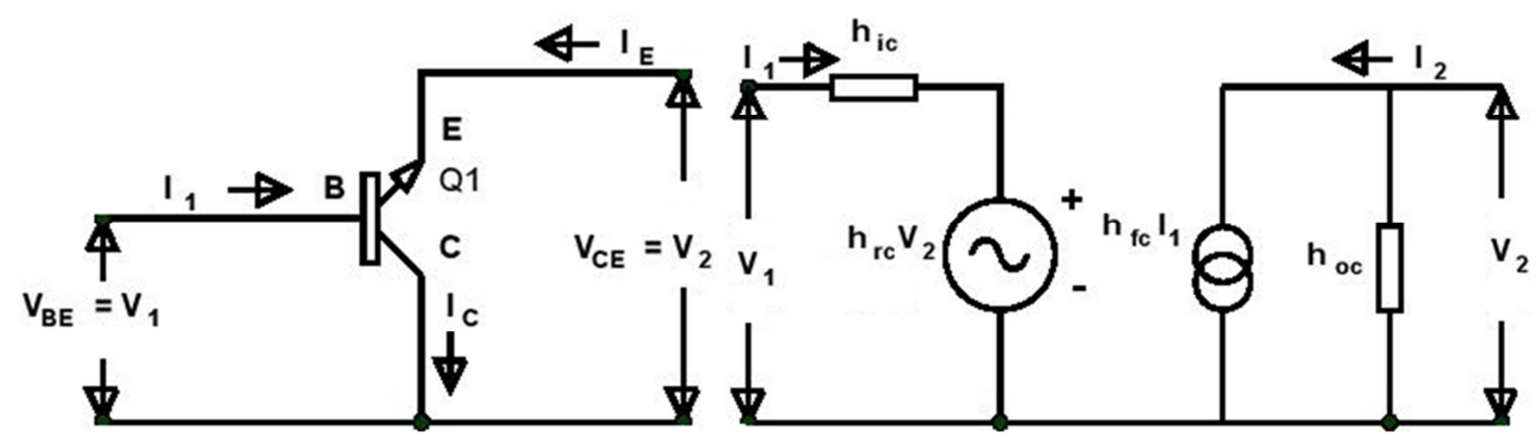

Figure 8. (a) common- collector configuration (b) h-parameters equivalent circuit of common-collector config.

\section{Results and Discussion}

From the analysis above, the derived h-parameter relating the four variables in the two-port network system can be conveniently represented for the three configuration of the transistor as follows:

1. Common- emitter (CE) configuration

$$
\begin{gathered}
V_{b e}=h_{i e} I_{b}+h_{r e} V_{c e} \\
I_{e}=h_{f e} I_{b}+h_{o e} V_{c e}
\end{gathered}
$$

2. Common-base $(\mathrm{CB})$ configuration

$$
\begin{gathered}
V_{e b}=h_{i b} I_{e}+h_{r b} V_{c b} \\
I_{c}=h_{f b} I_{e}+h_{o b} V_{c b}
\end{gathered}
$$

3. Common-collector (CC) configuration

$$
\begin{gathered}
V_{b c}=h_{i c} I_{b}+h_{r c} V_{e c} \\
I_{e}=h_{f c} I_{b}+h_{o c} V_{e c}
\end{gathered}
$$

The table below shows the h-parameters and their subscript designations for the three-circuit configuration.

The results of the analysis as shown in table 1 has adequately represent the performance quantities of the transistor h-parameter namely the matching impedances (input resistance and output admittance), the voltage gain and the current gain. From the generalized h-parameter representation, $\mathrm{h}_{11}$ relates the input voltage to input current at a zero output voltage and $h_{22}$ is the reverse of $h_{11}$ and relates the output current and voltage. The $h_{12}$ and $h_{21}$ are the gain quantities of the h-parameters. The reverse voltage gain relates the input and output voltages while the forward current gain relates the output and the input currents. 
Table 1. List of h-parameters with their designations.

\begin{tabular}{|c|c|c|c|c|}
\hline Terms & Numbered parameters & CB parameters & CE parameters & CC Parameters \\
\hline Input resistance & $\mathrm{h}_{11}$ & $\mathrm{~h}_{\mathrm{ib}}$ & $\mathrm{h}_{\mathrm{ie}}$ & $\mathrm{h}_{\mathrm{ic}}$ \\
\hline Reverse Voltage Gain & $\mathrm{h}_{12}$ & $\mathrm{~h}_{\mathrm{rb}}$ & $\mathrm{h}_{\mathrm{re}}$ & $\mathrm{h}_{\mathrm{rc}}$ \\
\hline Forward current again & $\mathrm{h}_{21}$ & $\mathrm{~h}_{\mathrm{fb}}$ & $\mathrm{h}_{\mathrm{fe}}$ & $\mathrm{h}_{\mathrm{fc}}$ \\
\hline
\end{tabular}

\section{Conclusion}

In this analysis, it has been sufficiently demonstrated that h-parameters for transistor amplifiers can be correctly realized using mathematical equations as opposed to the derivation of transistor operating conditions from its characteristics curve at a certain operating regions. From the results obtained, it has been shown that the h-parameter performance quantities of a small signal transistor can be derived fundamentally using the transistor two-port network system for the three configurations of the transistor amplifier. The results has also adequately represented the operation and actions of a small signal transistor in impedance matching and various transistor gains.

The outcomes of this study has concisely described the important components of the transistor h-parameter which are mainly the matching impedances namely the input resistance and output admittance; the gains namely the voltage gain and the current gain. These components are real numbers at audio frequencies and are easy to measure. The process and results is easy to comprehend even by those with little knowledge in mathematics. Therefore, this study can aid the teaching and understanding of students at all levels. Again, the process and outcome can be computerized to ease the rigors of transistor analysis.

\section{References}

[1] "Analysis of Small-signal Transistor Amplifiers," Routledge Taylor and Francis Group, 2018.

[2] Andy Collinson, "Transistor Hybrid Model," Circuit Exchange International, 2018.

[3] BrainKart, "Small Signal Low Frequency h- parameter Model," BrainKart Lectures, 2017- 2018.

[4] Charles H. E., "Electronic Amplifier," Delmar Publishers, Albany, New York, 1979.
[5] Robert B. and Louis N., "Electronic Devices and Circuit Theory," Prentice-Hall International, Inc. USA, 1996.

[6] Theraja A. K. and Theraja B. L., "A textbook of Technology," S. Chad \& Company Ltd, Raw Nagar, Nardeth, 1999.

[7] Lobna A. Said, Ahmed G Radwan, A. H. Madian and A. M. Soliman, "Two Port Network Analysis for three impedance based Oscillators," ResearchGate, December 2011. DOI: 10.1109/ICM.2011.6177421.

[8] Ahmed S. Elwakil and Muhammad Ali Al- Radhawi "All possible second-order four- impedance two-stage Colpitts oscillators," The Institution of Engineering and Technology Circuits, Devices \& Systems, Vol. 5, Iss. 3, pp.196-202, 2011. DOI: 10.1049 /iet- cds.2010.0201

[9] Ahmed S. Elwakil "Design of non- balanced cross-coupled oscillators with no matching Requirements," The Institution of Engineering and Technology Circuits, Devices \& Systems, 2010a, Vol. 4, Iss. 5, pp. 365-373 DOI: 10.1049/ietcds.2010.0030.

[10] Ahmed S. Elwakil, "Motivating two-port network analysis through elementary and advanced examples," International Journal of Electrical Engineering Education, 2010b.

[11] David B Haviland, "The Transistor in a Century of Electronics," Nobel Media, 2002.

[12] "The History of the Transistor," San José State University, Silicon Valley, \& Tornado Alley, USA.

[13] Michael Riordan, "The Lost History of the Transistor," IEEE Spectrum, 2004.

[14] Jose Tadeu Arantes, "Transistor that mimics neurons developed," 2017.

[15] Eneh I. I., "Principles of Electronic Devices and circuits," 1999. Rojoint Communication Ltd, Enugu Nigeria.

[16] Ruye Wang, "Small-Signal Model and H parameters," semiconductor Devices and Circuit 2017. 